\title{
Diseño Industrial, un hacer responsable con la sociedad
}

\author{
Paola Andrea Roa López ${ }^{1}$
}

Recibido: 04-08-2017

Aceptado: 11-10-2017

\section{RESUMEN}

El ser humano a través del tiempo ha modificado su medio natural a satisfacción de sus necesidades, esto ha traído consecuencias sociales, ambientales y económicas para el desarrollo social y humano. Disciplinas como el Diseño Industrial, cuyo Objetivo es la configuración y producción del medio objetual, deben desarrollar un hacer reflexivo frente a las evidentes consecuencias de una actividad poco consciente y desmedida en una sociedad en muchos casos consumista y egoísta. El siguiente es un artículo reflexivo sobre la responsabilidad social del hacer del Diseñador Industrial, dando a conocer un caso exitoso de impacto social.

Palabras clave: Diseño Industrial - Diseño Responsable - Diseño y Sociedad - Sociedad. 


\title{
Industrial Design, a responsible doing with society
}

\begin{abstract}
Through the time the human being had been modified his natural environment to benefit of his needs, this had brought social, environmental and economical consequences for the social and human development. The industrial design, which goal is the configuration and production of the object way, should develop a reflexive do in front of the clear consequences of an a little conscious activity in a society to many cases consumer and selfish . the following is a reflexive article about the social responsibility of the industrial designers do, giving to know a successful case of a social impact.
\end{abstract}

Keywords: Industrial Design - Responsible Design - Design and Society - Society

\begin{abstract}
Al principio de los tiempos, el ser humano era vulnerable a su entorno natural, sufriendo todo tipo de agresiones, principalmente de manera física, obligándolo a modificarlo para garantizar principalmente su supervivencia. Esta vulnerabilidad y necesidades, motivaron la modificación de factores como la materia prima que tenían a su alcance para la generación de objetos y utensilios con un fin, como es el caso del Homo habilis, que daba forma a las piedras golpeándolas hasta lograr herramientas de caza. Esta primera generación de objetos con un fin específico es señalada comúnmente como el inicio del Diseño Industrial, sin embargo es considerado de igual manera como un primer momento llamado preindustrial, en el cual se configuraron los primeros objetos de la humanidad sin un proceso preconcebido.
\end{abstract}

Con el paso de los siglos, el hombre a la medida de su desarrollo, ha aumentado considerablemente sus necesidades y expectativas en búsqueda de condiciones más cómodas de vida. Llega la Revolución 
Industrial y con ella un segundo momento, conocido como el industrial, que impulso la aparición del Diseño Industrial como disciplina y como configurador de objetos, con una función practica definida y estratégicamente planeada, unida a la función estética y con procesos industriales preconcebidos bajo el modelo seriado, este segundo momento generó las bases de la disciplina de lo que hoy se conoce como Diseño Industrial.

La llegada de la revolución industrial permitió avances significativos en la sociedad como producir más en menos tiempo y con menos esfuerzo, generar más infraestructura, más tecnología, reducción de los costos de fabricación, de los precios de los productos, aparece la división del trabajo y la especialización, cada uno se ocupa de una tarea en especial, una persona idea, otra produce, lo que marca la diferencia entre la labor artesanal desarrollada hasta el momento y el proceso industrial actual, de igual manera se aplica energía eléctrica en la industria, se mejoran los medios de comunicación y transporte, entre muchos más.

La Revolución Industrial trajo de igual forma consecuencias no medidas por sus propulsores, como el dominio del capitalismo, en el cual los comerciantes, industriales y productores iniciaron la monopolización del comercio a gran escala, con el fin de realizar inversiones de capital con miras a la competencia por los mercados de consumo y el trabajo asalariado, la revolución Industrial incrementó las horas de trabajo y las tareas repetitivas, trayendo consigo familias disgregadas y obreros con conocidas enfermedades dadas por su labor, aparecen desigualdades y contrastes sociales entre el sector obrero y el sector burgués lo que trae consigo un desenfrenado aumento en la desigualdad y concentración de riqueza, gracias a la revolución Industrial las personas empiezan a pensar que el comprar hace feliz, idea que dio inicio al consumismo desmedido en el que hoy se encuentra inmersa la sociedad y uno de los motivos principales de la contaminación ambiental, dado que después de la Revolución Industrial, la industria creció indiscriminadamente y con ella las emisiones al medio natural, contaminando los recursos naturales, fuentes de la vida humana, como lo son el aire, el agua y el suelo. Para el Año 2004 las emisiones de carbono llegaron al record histórico de 6.800 millones de toneladas globalmente. (Terra Ecología y Práctica, 2004). Desde la década de 1950, ver la figura 1, las emisiones anuales se han cuadriplicado, tres cuartas partes de las emisiones globales de carbono provienen de combustibles fósiles como el petróleo y el gas natural, El resto son, mayoritariamente, consecuencia de la deforestación. De 
los cuatro principales sectores que contribuyen a estas emisiones, la generación de electricidad es responsable de la mayor parte: un 35\%. El transporte y los procesos industriales son responsables de un $20 \%$ cada uno, mientras que el $25 \%$ restante proviene de edificios residenciales y comerciales. (Terra Ecología y Práctica, 2004)

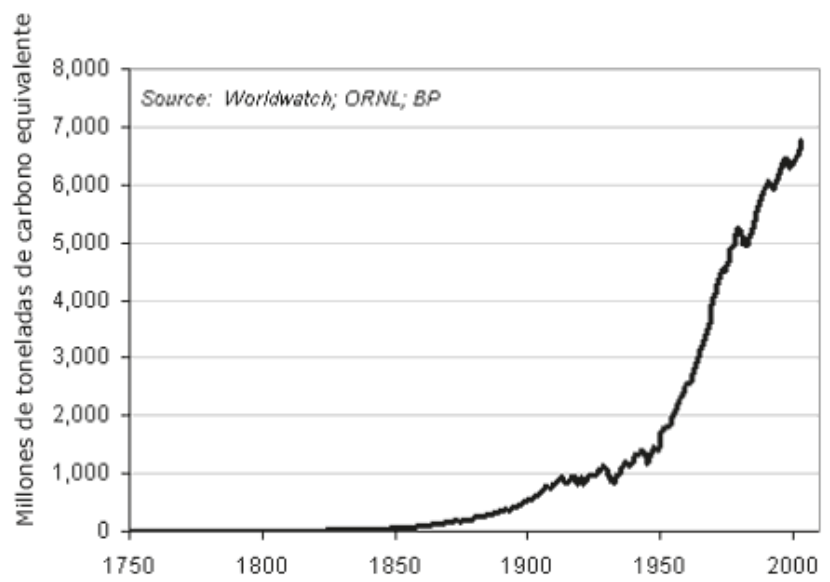

Figura 1. Emisiones Globales Generadas por la combustión de Combustibles Fósiles, 1751, 2003. (Terra Ecología y Práctica, 2004)

Actualmente la sociedad se encuentra en el tercer momento del Diseño Industrial, precedido de algunas de las consecuencias de la revolución industrial, éste es el momento de la posmodernidad, que Jean Baudrillard define como "Una época en donde se pierde la narrativa dominante, afirmando que nadamos en un mar de lenguajes privados". (Foster \& Baudrillard, 2008). Estos lenguajes modifican el resultado del Diseño Industrial llamado objeto, dado que estos ya no son buscados por usuarios y compradores por su función, al contrario, el usuario o comprador le asigna una función al objeto o artefacto en medio de una sociedad de consumo, tema que merece una reflexión cuidadosa dado que el Diseño Industrial ésta ligado al desarrollo de las corrientes de la producción industrial y de esta forma puede elevar el nivel de vida de la humanidad, llegando a ser generador de falsas necesidades sociales y aún más dentro del consumismo, movido principalmente por el pensar de la revolución Industrial: comprar te hace feliz, como Indica Karin Thomas: 


\begin{abstract}
"Mediante la publicidad y el diseño industrial se le impone al hombre un gusto conformista. El asentimiento de la gran masa a esta determinación del gusto, que se exterioriza en el placer adquisitivo, no comporta una valoración efectiva y aquiescente de la función del objeto de consumo, sino un auto doblegamiento a la coacción social". El diseño en la forma practicada por el capitalismo sirve a la manipulación, sin ventaja para el desenvolvimiento de la personalidad". (Thomas, 1978)
\end{abstract}

La condición actual del mundo, su contaminación, sobrepoblación, consumismo, la industrialización y una sociedad que compra objetos buscando en ellos la felicidad o suplir necesidades de prestigio, encantamiento o distinción, promueven un llamado a la ética de profesionales como los Diseñadores Industriales cuyo objetivo es generar la cultura material y objetual de la sociedad.

El Diseño Industrial según el ICSID (Consejo Internacional de las sociedades de diseño industrial) es:

"Disciplina y actividad creadora que tiene como tarea
determinar las propiedades formales de los objetos que se van
a reproducir industrialmente, entendiendo por propiedades
formales no sólo las características exteriores sinolas espaciales
también, así como las relaciones estructurales que permiten a
un objeto industrial convertirse en una unidad coherente y de
uso", (ICSID, 2010).

Es importante destacar que el ICSID hace énfasis en que no necesariamente el Diseño industrial debe producir objetos en serie, si no que se le denomina así por construir objetos mecánicamente para usuarios finales y grupos sociales. Dada la actualidad, el hacer del Diseñador Industrial debe estar enfocado en satisfacer las necesidades reales de la sociedad y no pretender generar nuevas necesidades, como es el caso de productos cuya producción seriada inundan el mercado, consumen energía, materias primas, mano de obra, recursos naturales, aire, espacio, tiempo en su producción y no son más que elementos decorativos, elevadores de ego u objetos tan carentes de sentido y finalidad que terminan aumentando los botaderos de las grandes ciudades o alimentando necesidades sin necesidad de personas que buscan llenar falencias emocionales, físicas o psicológicas entre muchas más, con la adquisición de productos. 
"Mediar dialécticamente entre necesidades y objetos, entre producción y consumo. Por lo general, el diseñador está demasiado inmerso en la rutina de su profesión y no llega a intuir la incidencia social efectiva de su actividad. Ello se desprende de la concepción tan difundida de un diseño industrial entendido como intervención absolutamente aislada, como una "prestación", un "servicio" a la industria". (Maldonado, 1977)

El código de Ética Profesional del Diseñador Industrial de la ICSID, promueve un hacer responsable, como lo indica en su Artículo II, donde exhorta a los diseñadores a tener conciencia de las necesidades de todos los usuarios potenciales, incluidos aquellos con diferentes capacidades físicas y en especial a las personas de la tercera edad, logrando así, humanizar la tecnología. De igual manera el Artículo III indica la necesidad de proteger el Ecosistema Terráqueo, dado que los intereses de las generaciones presentes y futuras solo se pueden proteger si el ecosistema terrestre se salvaguarda.

Si el Diseñador Industrial se detiene a pensar en un hacer responsable y reflexiona en su papel como generador de la cultura material que le rodea, será un paso adelante en el Desarrollo Humano, que Amartya Sen, define como un enfoque centrado en las personas, más que el crecimiento económico. "Lograr el Desarrollo del potencial humano, a través de estrategias que permiten a las personas y comunidades realizar sus visiones de desarrollo, superar las condiciones de su marginación, e integrarse plenamente en sus respectivas sociedades". (Sen, 2000).

\section{Según Hans Gugelot "El objetivo del diseñador debe ser crear buenos bienes de consumo que puedan ser producidos y no buenos bienes de producción que deban ser consumidos". (Gay \& Samar, 2007)}

Siendo coherentes con las palabras Hans Gugelot de crear buenos bienes de consumo que puedan ser producidos y un enfoque centrado en las personas como lo indica Amartya Sen, se detecta en Colombia y más específicamente en la Ciudad de Cali, una oportunidad de Diseñar y producir objetos que dignifiquen, valoren y engrandezcan con su uso a una población, para este caso en condición de discapacidad (PcD), que según el Ministerio de Salud y Protección Social y el censo del DANE 2011, son 978.218 personas, es decir el 2,1\% la población en Colombia para 
2012 se encontraba en condiciones limitadas, con una calidad de vida disminuida, ver Figura 2. En Cali el 6,4\% de sus habitantes pertenecen a la PCD, de los 2.269.630 habitantes que tenía la ciudad para 2011, 145.256 sufre de algún tipo de discapacidad, de estos 7.764 son niños y adolescentes de los cuales 1.826 tienen problemas de movilidad en brazos, manos o piernas con $19 \%$, le siguen las alteraciones del sistema nervioso, con el $18 \%$ y limitaciones visuales con el $17 \%$, pero la cifra más preocupante es que el $30 \%$ de estos (43.500) no tiene afiliación alguna al sistema de seguridad social (DANE, 2010), lo que reduce la posibilidad de terapia para su rehabilitación, generando de esta forma una barrera para un trato digno en su inclusión social.

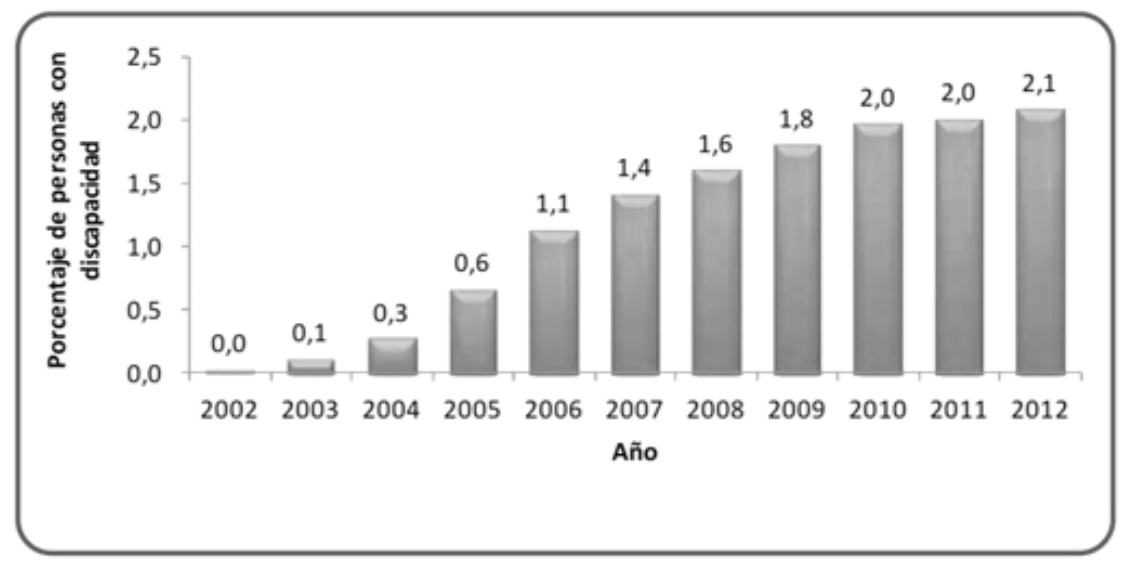

Figura 2. Porcentaje de Personas registradas por año. (Ministerio de Salud y Protección Social., 2014)

Basados en las estadísticas del crecimiento de las PcD, sus necesidades objetuales para el desarrollo de terapias, la continua búsqueda de mejorar sus condiciones de vida y el valor de humanizar la tecnología desde el Diseño Industrial, permiten a la Fundación Academia de Dibujo Profesional (FADP) por medio de los estudiantes de Diseño Industrial convertirse en medio de Inclusión Social de las PcD en la región, sensibilizando al estudiante en su hacer profesional con responsabilidad social y permitiéndole al mismo tiempo ver su profesión como una herramienta social en el desarrollo de artefactos que faciliten las terapias y rehabilitación en adultos y niños en condición de discapacidad, ofreciéndoles de esta forma el derecho a vivir de una manera digna e independientemente y ser Incluidos en la comunidad como lo indica el artículo 19 de los derechos de las PCD en Colombia. 
Las herramientas diseñadas y producidas son donadas a fundaciones en la región a las cuales asisten las PCD de bajos recursos de la ciudad en búsqueda de ayuda y un alivio para su condición. Es necesario dar a conocer que aunque estas instituciones o fundaciones reciben a los niños o adultos discapacitados con toda la disposición, comprensión, acompañamiento y amor que estas personas y especialmente los niños buscan, muchas veces sus metas y alcances se ven frustrados por la falta de recursos económicos que les permitan adquirir las herramientas técnicas para el desarrollo de terapias o procesos que lleven a la persona en condición de discapacidad a alcanzar sus objetivos o sueños como lograr el movimiento de una de sus extremidades, aprender a leer, escribir, contar, conocer colores, texturas, partes del cuerpo o valores, entre otros como alivianar el trabajo físico del personal que asiste a las Pcd.

Anualmente se donan aproximadamente 30 herramientas a las fundaciones, que evidencian en sus alcances de aplicación la estrategia cognitiva, metodológica y conceptual de los estudiantes de Diseño Industrial, de igual manera dichas herramientas reflejan su sentido funcional, formal y de uso, que sumando al hacer ético y responsable con la sociedad de las nuevas generaciones de Diseñadores, han logrado facilitar el proceso de terapia de la población en situación de discapacidad de la región, facilitando de esta forma los procesos en búsqueda de la igualdad y no discriminación, planteado en el artículo 5 de los derechos de las PcD. (Ministerio de Salud y Protección Social, 2013).

A continuación se relacionan algunas de las herramientas diseñadas, producidas y donadas por los estudiantes de Diseño Industrial a las fundaciones que trabajan con personas en condición de discapacidad de bajos recursos en la ciudad de Cali. 


\section{Muestra de Herramientas Diseñadas, Producidas y Donadas}

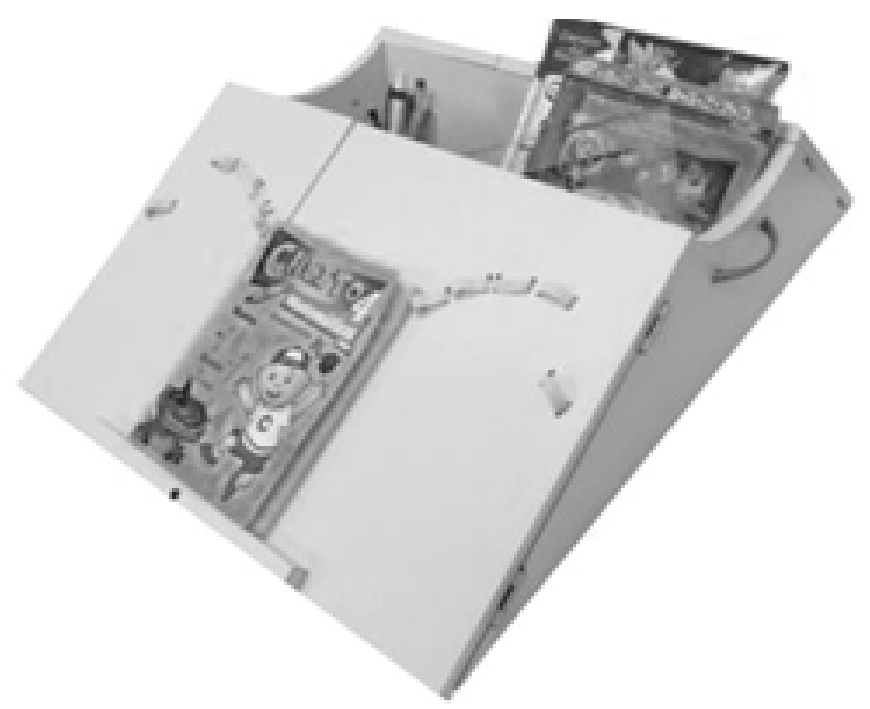

Imágen 1. Herramienta para la motricidad fina, (Murillo, Cedeño \& Rosas. 2011).

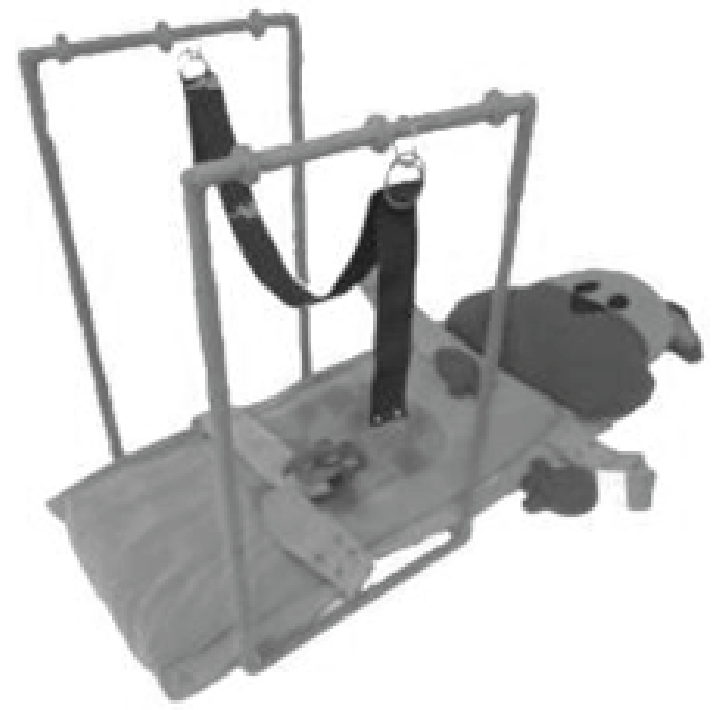

Imágen 2. Camilla para terapia en brazos y piernas, (Bravo, Crono \& Velazco. 2011). 


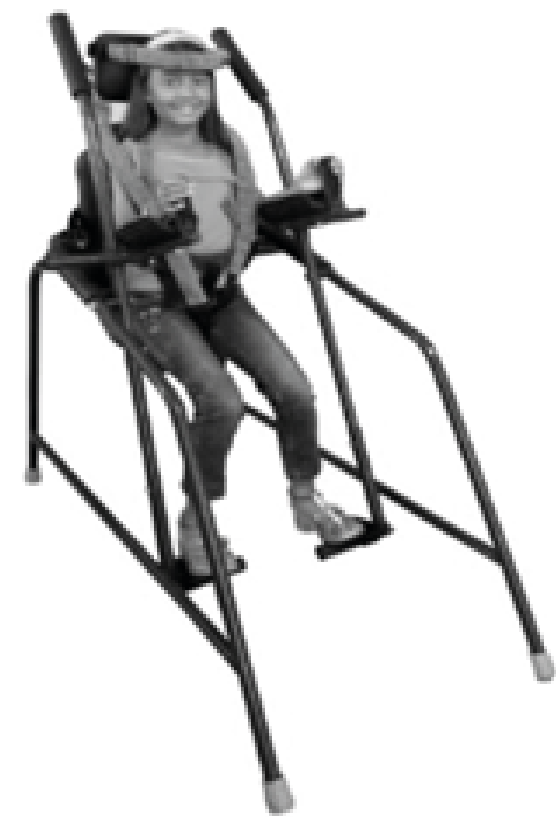

Imágen 3. Máquina de Ejercitación para niños con cuadriplejia y hemiplejia (Corrales, Garzón \& Álvarez. 2011)

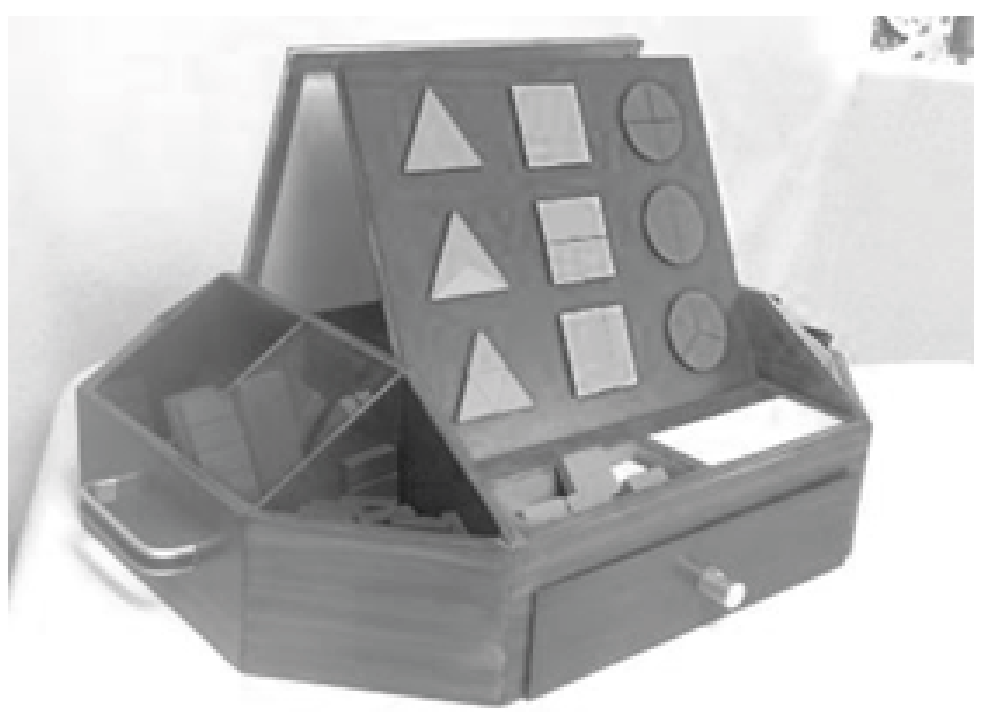

Imágen 4. Herramientas para invidentes y discapacidad mental moderada. (Rengifo, Charry \& Gutiérrez. 2013). 


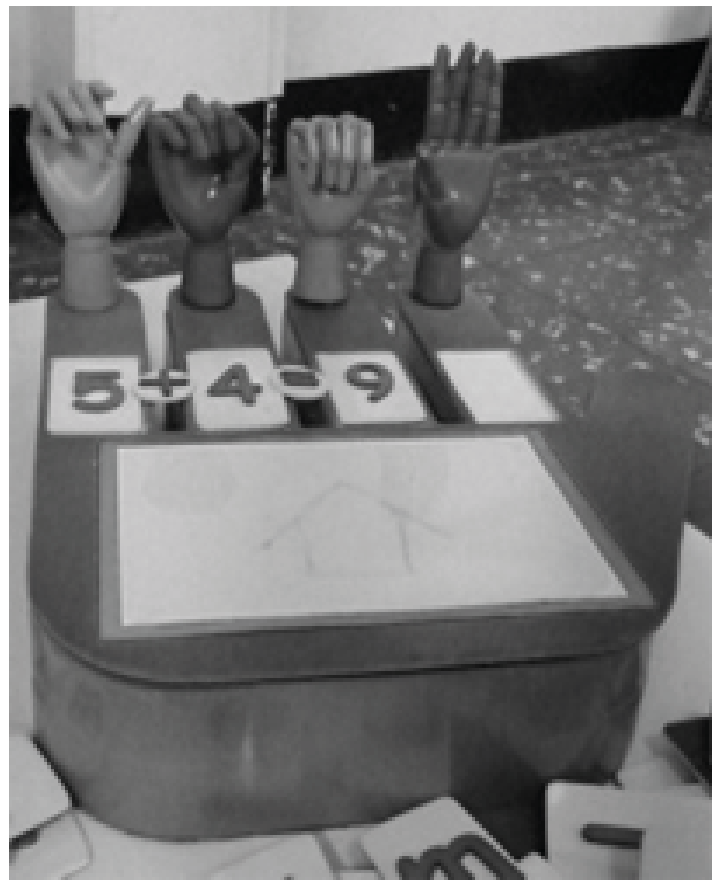

Imágen 5. Herramienta para la enseñanza de español y lenguaje de señas para niños sordos. (García, La Torre \& Ocampo. 2014)

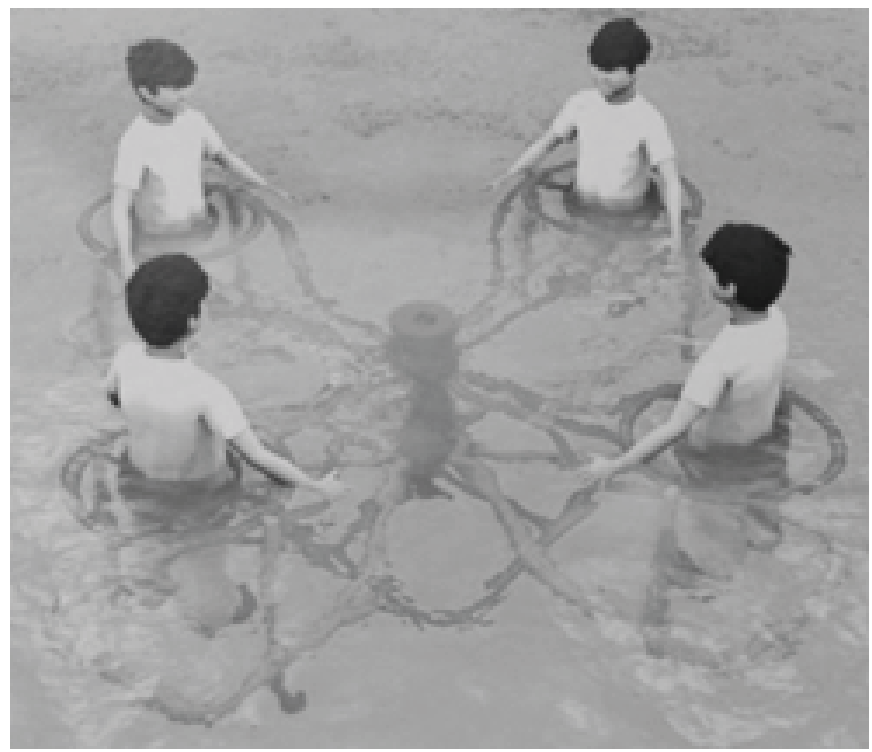

Imágen 6. Estimulación de niños cuadripléjicos o con discapacidad cognitiva severa en el agua. (Castro, González \& Montes. 2014). 
Para el diseño, producción y donación de las herramientas para niños en condición de discapacidad, se desarrolló la metodología descriptivo analítica, la cual consta de tres fases:

\section{Fase conceptual}

Los estudiantes investigan y conocen los conceptos de discapacidad, diseño social, enfermedades incapacitantes y herramientas de terapia, con el objetivo de obtener el conocimiento necesario para enfrentarse al proyecto.

\section{Fase Observatoria}

Los estudiantes identifican una fundación o institución de bajos recursos de la ciudad, que trabaje con adultos y especialmente con niños discapacitados, para la cual el proyecto a realizar genere un impacto positivo en el proceso de terapias, con el objetivo de facilitar su inclusión social y mejorar su calidad de vida. En esta fase los estudiantes visitan la fundación para detectar en ellas la carencia de herramientas y en común acuerdo con psicólogos, fisioterapeutas o directivas, determinar el proyecto a desarrollar.

\section{Fase Práctica}

Diseñan, producen y donan la herramienta desarrollada que suplirá una necesidad de terapia o enseñanza en las fundaciones o instituciones que trabajan con la población en discapacidad de bajos recursos, todo bajo la asesoría docente. (Fase Final - Productiva).

La cantidad de proyectos diseñados y donados a las fundaciones al momento suman 86 prototipos en 46 fundaciones en la región.

El proyecto desarrollado con las $P C D$, es una muestra del valor de un hacer responsable desde el Diseño Industrial, viéndolo como una profesión que busca mejorar su calidad de vida, con la satisfacción de sus necesidades objetuales y permitiendo generar en los estudiantes un hacer reflexivo y responsable con la sociedad y el medio ambiente, siendo participe de un cambio de pensamiento frente a la realidad comercial, consumista y ambiental en la que la sociedad se encuentra actualmente.

Con el desarrollo del proyecto y enfrentando a los estudiantes a una realidad social, se evidencia en ellos un cambio en su pensamiento y responsabilidad social bajo su profesión. A continuación se dan a conocer algunos de sus comentarios al respecto: 
"Es gratificante saber que por medio de tu esfuerzo y aprendizaje puedes ayudar a esas personas que erradamente son llamadas discapacitados. Como experiencia me queda la alegría en los rostros de padres, pacientes y demás personas que se comprometen día a día con su labor". (Bravo. 2011).

"El haber tenido la oportunidad de trabajar con niños en condición de discapacidad ha sido muy gratificante, ya que he logrado sensibilizarme a cerca de las diferentes situaciones que afectan a los niños en estas condiciones, dado que se ven enfrentados a un entorno que genera obstáculos y que imposibilitan el libre desarrollo de sus actividades diarias. Este proyecto nos llena de mucha satisfacción, pues sabemos que estamos aportando un granito de arena". (Argoti. 2015)

"Esmuysatisfactoriopodercontribuirmedianteunconocimiento en el desarrollo terapéutico de los niños discapacitados, lo más agradable de todo el proceso fue aprender a ver el mundo entendiendo que estos niños realmente son más valientes de lo que creemos, más fuertes de lo que parecen y más capaces que lo que pensamos. Doy gracias a la Fundación Academia de Dibujo Profesional y ante todo a nuestra asesora Paola Roa por permitirnos ser parte este proyecto". (González. 2014)

"Poder ayudar sin esperar nada a cambio más que una sonrisa ha sido de verdad, una excelente experiencia, saber que uno puede aportar en el desarrollo de otra persona con el diseño de un artefacto facilitador es lo que me hace creer aún más en el Diseño Industrial como una herramienta que puede generar inclusión. Gracias al acompañamiento brindado por la Fundación Academia de Dibujo Profesional puedo afirmar que logramos sacar adelante un proyecto social muy gratificante". (Montes. 2014)

Autores como Gui Bonsiepe y André Ricard comparten la finalidad del Diseño Industrial viéndolo como la producción de objetos que responden a demandas, necesidades, deseos o aspiraciones de la sociedad, teniendo en cuenta además de las características exteriores, las relaciones funcionales y estructurales que hacen del objeto un todo coherente con dichas necesidades. 
"El arte posee una justificación en sí mismo mientras que el diseño se fundamenta en el uso social del objeto". (Bonsiepe, 1975)

"El diseñador no debe buscar la exteriorización de su personalidad en el diseño, sino la función que el objeto diseñado cumple en la sociedad y el uso que ésta hace de ese objeto". (Bonsiepe, 1975).

"El Diseño no trata la forma por la forma, sino que la define en función de la utilidad que ésta ha de posibilitar. No intenta adornar a los nuevos artefactos tecnológicos, ni maquillar a los objetos tradicionales, sino que pretende dotarlos de aquella peculiar configuración que habrá de permitirles mejorar su función útil, es decir: su servicio y su relación con el hombre. La forma es el medio por el cual se hace posible la función útil de lo material.". (Ricard, 1982)

\title{
Referencias bibliográficas
}

\author{
Alvarado, G., Roa, P., \& Zuleta, D. (2016). Formación en Diseño Industrial: Una propuesta \\ metodológica coherente con el desarrollo sostenible. Revista Interamericana De \\ Educación, Pedagogía Y Estudios Culturales, 163. \\ Bonsiepe, G. (1975). Diseño Industrial Artefacto y Proyecto. Ibid. \\ Carvajal, M., \& Zapata, A. (2016). Diseñar con conciencia. Revista Interamericana De \\ Educación, Pedagogía Y Estudios Culturales, 205. \\ Ceben. (s.f.). Cooperación, Conocimiento y Desarrollo. Recuperado el 21 de agosto de \\ 2015, de http://ccd-ckd.cebem.org/pages/tophum/index_esp.php \\ DANE. (2010). Dirección de Censo y demografia. Cali. \\ FADP. (2009). Proyecto Educativo Institucional 2009-2013. Cali. \\ Foster, H., \& Baudrillard, J. (2008). La posmodernidad. Kairós. \\ Gay, A., \& Samar, L. (2007). El Diseño Industrial en la Historia. Argentina: Tec.
}


ICSID. (2010). Codigo de Ética Profesional.

Maldonado, T. (1977). El Diseño Reconsiderado. Barceona: Gustavo Gili.

Ministerio de Salud y Protección Social. (2013). Informe inicial sobre la implementación de la convención sobre los derechos de las PCD en Colombia. Colombia.

Ministerio de Salud y Protección Social. (2014). Boletín 1 Observatorio Nacional de Discapacidad.

Ricard, A. (1982). Diseño ¿Por qué? Barcelona: Gustavo Gili.

Roa, P. A. (2013). El Diseño Industrial en la satisfacción de necesidades. Obtenido de http://www.fadp.edu.co/publicaciones/proyinvind-07.pdf

Sen, A. (2000). Desarrollo y Liberta. Buenos Aires: Planeta.

Terra Ecología y Práctica. (4 de Noviembre de 2004). Las emisiones de carbono llegan a un récord histórico. Recuperado el 2 de Agosto de 2015, de http://www.terra.org/ categorias/articulos/las-emisiones-de-carbono-llegan-un-record-historico

Thomas, K. (1978). Diccionario del Arte Actual. Barcelona: Labor. 